\title{
EXTREME POINTS OF POLAR CONVEX SETS
}

\section{R. R. PHELPS ${ }^{1}$}

1. Introduction. Suppose that $E$ is a normed linear space and that $f \in E^{*},\|f\| \leqq 1$. There is a closed, convex subset $E_{f}$ of $E$, intimately related to $f$, which is defined as follows: $E_{f}=\{x \in E:\|x\|-f(x) \leqq 1\}$. R. C. Buck [2] has proved (among other things) that $f$ is an extreme point of the unit ball $U^{*}$ of $E^{*}$ if and only if $E=E_{f}-E_{f}$, i.e., $f$ cannot be expressed as the midpoint of a line segment in $U^{*}$ if and only if every point of $E$ is of the form $u-v$, where $u$ and $v$ are in $E_{f}$. Consider this result from a different viewpoint: Letting

$$
C=\{x \in E:\|x\| \leqq 1\},
$$

the theorem gives, in terms of $f$ and the norm in $E$ (i.e., the gauge functional of $C$ ), a necessary and sufficient condition that an element $f$ in the polar $C^{0}$ of $C$ in $E^{*}$ be an extreme point of $C^{0}$. Note that the set $C$ is closed, convex, symmetric about the origin, and has nonempty interior. The main purpose of the present note is to give an elementary proof of a similar characterization of the extreme points of $C^{0}$ without making any assumptions about $C$ other than it be a convex subset, containing the origin, of some linear space $F$. In order to consider the polar of $C$ we naturally require that $F$ be in duality with another space $G$; the duality inherent in our formulation will enable us to deduce a dual version of Buck's theorem.

2. The main theorems. Using the notation of [1], let $F$ and $G$ be real or complex linear spaces in duality under $\langle$,$\rangle . (In all that follows,$ the symbol $\langle$,$\rangle will stand for \operatorname{Re}\langle$,$\rangle in case F$ and $G$ are over the complex field.) Let $C$ be a convex subset of $F$ which contains the origin $\phi$, and let $p$ be the gauge functional defined by $C$; for $x \in F$, $p(x)=\inf \left\{\lambda>0: \lambda^{-1} x \in C\right\}$. Note that $0 \leqq p(x) \leqq \infty$ (the latter equality holding if and only if $\lambda^{-1} x \notin C$ for all $\left.\lambda>0\right), p(x+y) \leqq p(x)+p(y)$ for all $x, y$ in $F, p(\alpha x)=\alpha p(x)$ if $\alpha>0$, and $p(x) \leqq 1$ if $x \in C$. Let $C^{0}$ be the polar of $C$ in $G$, i.e., $C^{0}=\{y \in G:\langle x, y\rangle \leqq 1$ for all $x \in C\}$. It follows from the definitions that if $y \in C^{0}$ and $x \in F$, then $\langle x, y\rangle \leqq p(x)$. Supposing $y \in C^{0}$, let $F_{y}=\{x \in F: p(x)-\langle x, y\rangle \leqq 1\}$; the subadditivity of $p$ implies that $F_{y}$ is convex: The topology $\sigma(F, G)$ on $F$ is locally convex, and hence [3, p. 22] a convex subset $K$ of $F$ is not $\sigma(F, G)$-dense

Presented to the Society, April 23, 1960; received by the editors April 18, 1960.

${ }^{1}$ Work on this paper was performed, in part, while the author was a National Science Foundation Postdoctoral Fellow. 
in $F$ if and only if there exists a $z \in G \sim\{\phi\}$ and $b>0$ such that $\langle x, z\rangle$ $\leqq b$ for all $x$ in $K$. By considering $b^{-1} z$ instead of $z$, we may, if we wish, assume $b=1$.

Theorem 1. A point $y \in C^{0}$ is an extreme point of $C^{0}$ if and only if $F_{y}-F_{y}$ is $\sigma(F, G)$-dense in $F$.

Proof. Since $F_{y}-F_{y}$ is convex, if it is not $\sigma(F, G)$-dense in $F$, there exists a $z \in G \sim\{\phi\}$ as above. Since $x \in F_{y}-F_{y}$ if and only if $-x \in F_{y}$ $-F_{y}$, and since $F_{y} \subset F_{y}-F_{y}$, we have $|\langle x, z\rangle| \leqq 1$ for all $x \in F_{y}$. We will show that the points $y \pm z$ are in $C^{0}$; since $y=2^{-1}(y+z)+2^{-1}(y-z)$, we can then conclude that $y$ is not an extreme point of $C^{0}$. We must show, then, that $\langle x, y \pm z\rangle \leqq 1$ for all $x \in C$. Take $x \in C$ (so $p(x) \leqq 1<\infty)$ and let $\alpha=p(x)-\langle x, y\rangle$. If $\alpha=0$, then $\lambda x \in F_{y}$ for all $\lambda>0$; since $|\langle\bar{x}, z\rangle| \leqq \lambda^{-1}$, we have $\langle x, z\rangle=0$ and hence $\langle x, y \pm z\rangle=\langle x, y\rangle \leqq 1$. If $\alpha>0$, then $1=\alpha^{-1} \alpha=p\left(\alpha^{-1} x\right)-\left\langle\alpha^{-1} x, y\right\rangle$, so $\alpha^{-1} x \in F_{y}$. Hence $\left\langle\alpha^{-1} x, z\right\rangle$ $\leqq 1$ or $\pm\langle x, z\rangle \leqq \alpha=p(x)-\langle x, y\rangle$ and therefore $\langle x, y \pm z\rangle \leqq p(x) \leqq 1$. Thus, $y \pm z \in C^{0}$ and we have finished one part of the proof. To prove the converse, suppose that $y$ is not an extreme point of $C^{0}$. There exist $u$ and $v$ in $C^{0}$ such that $y=2^{-1}(u+v)$; equivalently, there exists $z \in G \sim\{\phi\}$ such that $y \pm z \in C^{0}$ (take $z=v-y$, say). Thus, if $x \in F$, we have $\langle x, y \pm z\rangle \leqq p(x)$ or $\langle x, z\rangle \leqq p(x)-\langle x, y\rangle$. Therefore $\langle x, z\rangle \leqq 1$ for all $x \in F_{y}$, and hence $\langle x, z\rangle \leqq 2$ for all $x \in F_{y}-F_{y}$, which shows that the latter is not $\sigma(F, G)$-dense in $F$ and completes the proof.

Following Buck's example, we will now use Theorem 1 to prove a more general result. If $M$ is a linear subspace of $F$ (not necessarily closed) let $M^{\perp}=\{y \in G:\langle x, y\rangle=0$ for all $x \in M\}$ (this is simply $M^{0}$ ). With $C$ as before, we can characterize the extreme points of $C^{0} \cap M^{\perp}$ as follows:

THEOREM 2. $A$ point $y \in C^{0} \cap M^{\perp}$ is an extreme point of $C^{0} \cap M^{\perp}$ if and only if the set $F_{y}-F_{y}+M$ is $\sigma(F, G)$-dense in $F$.

Proof. Let $C_{1}=C+M$ and define $p_{1}, C_{1}^{0}$ and $F_{y}^{1}$ (for $y \in C^{0}$ ) in the obvious way. By Theorem 1, $y$ is an extreme point of $C_{1}^{0}$ if and only if $F_{y}^{1}-F_{\nu}^{1}$ is $\sigma(F, G)$-dense in $F$. The proof is completed by showing that $C_{1}^{0}=C^{0} \cap M^{\perp}$, and that for all $y$ in $C_{1}^{0}, F_{y}-F_{y}+M$ is a $\sigma(F, G)$ dense subset of $F_{y}^{1}-F_{\nu}^{1}$. The former fact follows easily from the definitions. The latter remark may be proved as follows: Note first that for $x \in F, p_{1}(x)=\inf \{p(x+m): m \in M\}$. Since $p(x+m) \leqq p(x)$, we have $F_{y}+M \subset F_{y}^{1}$ and hence $F_{y}-F_{y}+M \subset F_{y}^{1}-F_{y}^{1}$. If $x \in F_{y}^{1}$, let $u_{n}=\left(1-n^{-1}\right) x$. Then $p_{1}\left(u_{n}\right)-\left\langle u_{n}, y\right\rangle<1$ so there exists $m_{n} \in M$ such that $p\left(u_{n}+m_{n}\right)<1+\left\langle u_{n}, y\right\rangle=1+\left\langle u_{n}+m_{n}, y\right\rangle$ and therefore $u_{n}+m_{n}$ $\in F_{y}$. Now, if $x-x^{\prime} \in F_{y}^{1}-F_{y}^{1}$, define $u_{n}, u_{n}^{\prime}$ and $m_{n}, m_{n}^{\prime}$ as above; 
then $u_{n}+m_{n}-\left(u_{n}^{\prime}+m_{n}^{\prime}\right) \in F_{y}-F_{y}$ and hence $u_{n}-u_{n}^{\prime} \in F_{y}-F_{y}+M$. Since $u_{n}-u_{n}^{\prime}$ clearly converges to $x-x^{\prime}$ in the $\sigma(F, G)$ topology, this completes the proof.

If $E$ is a topological vector space, then it is in duality with $E^{*}$ (the space of all continuous linear functionals on $E$ ) in the obvious way: $\langle x, f\rangle=f(x)$ for all $x \in E, f \in E^{*}$. (In keeping with our convention we will assume that $f(x)$ stands for $(\operatorname{Re} f)(x)$ if $E$ is complex.)

Theorem 3. Suppose that $E$ is a topological vector space, and that the convex set $C$ contains the origin in its interior. Then, for any subspace $M$, we have $E=E_{f}-E_{f}+M$ if (and only if) $f$ is an extreme point of $C^{0} \cap M^{\perp}$.

Proof. By Theorem 2, the "only if" statement is obvious, while, if $f$ is an extreme point of $C^{0} \cap M^{\perp}$, then $E_{f}-E_{f}+M$ is $\sigma\left(E, E^{*}\right)$-dense in $E$. Let $C^{\prime}=2^{-1}[C \cap(-C)]$; then $\phi \in$ int $C^{\prime}$, and if $x \in C^{\prime}$ we have $\pm 2 x \in C$ so that $x \in E_{f}$. Thus, $E_{f}$ (and therefore $E_{f}-E_{f}+M$ ) has nonempty interior. If there exists $u \in E \sim\left(E_{f}-E_{f}+M\right)$, then there exists [3, p. 22] a $g \in E^{*} \sim\{\phi\}$ such that $g(x) \leqq g(u)$ for all $x$ in $E_{f}-E_{f}+M$, contradicting the $\sigma\left(E, E^{*}\right)$-density of the latter. Thus, $E=E_{f}-E_{f}+M$, which proves the theorem.

It is worth noting that we have not required either $C$ or $M$ to be closed.

3. Applications to normed spaces. Suppose that $E$ is a (real or complex) normed linear space. Denote its unit ball by $U$, and let $U^{*}$ denote the unit ball of $E^{*}$. The following is an immediate corollary of Theorem 3.

Theorem 4 (R. C. Buck). Let $M$ be a subspace of $E$ and suppose $f \in U^{*} \cap M^{\perp}$. Then $f$ is an extreme point of $U^{*} \cap M^{\perp}$ if and only if $E=E_{f}-E_{f}+M$.

Note that the above theorem actually characterizes the extreme points of the unit ball of $(E / M)^{*}$. The following is a dual version of this theorem.

TheOREM 5. Let $M$ be a closed subspace of $E$ and suppose $x \in U \cap M$. Then $x$ is an extreme point of $U \cap M$ if and only if $E_{x}^{*}-E_{x}^{*}+M^{\perp}$ is weak*-dense in $E^{*}$.

Proof. This follows from Theorem 2 upon recalling that the weak* topology in $E^{*}$ is the $\sigma\left(E^{*}, E\right)$ topology, and observing that since $M$ is closed, it is the annihilator in $E$ of $M^{\perp} \subset E^{*}$.

Obviously, the above theorems characterize the extreme points of $U^{*}$ or $U$ by taking $M=\{\phi\}$ or $M=E$, respectively. 
4. Remarks. A natural question arises with respect to Theorem 5 (taking $M=E$ ): If $x$ is an extreme point of $U$, is it possible to conclude that $E_{x}^{*}-E_{x}^{*}$ actually equals $E^{*}$ ? Letting $Q$ be the natural embedding of $E$ into $E^{* *}$, it is clear that $E_{x}^{*}=E_{Q x}^{*}$, so (by virtue of Theorem 4) this is equivalent to the question: Must the image $Q x$ of an extreme point $x \in U$ be an extreme point of $U^{* *}$ ? We will exhibit a normed space for which the answer to this is negative, but the question is still open for complete normed (i.e., Banach) spaces. Suppose that $E$ is a normed linear space with unit ball $U$, and suppose $\hat{E}$ is the completion of $E$, with unit ball 0 . If there exists an $x \in U$ which is an extreme point of $U$ but not of $U$, then we see (by using the facts that $E^{*}=\hat{E}^{*}, E^{* *}=\hat{E}^{* *}$ and $\left.\overline{Q E}=Q(\hat{E})\right)$ that $Q x$ is not an extreme point of $Q U=\bar{Q} \bar{U} \subset U^{* *}$, and hence is not an extreme point of $U^{* *}$. The problem, then, is to find a space $E$ containing a point $x$ with the above properties. Define a new norm on the (real) space $l_{2}$ as follows: The new unit ball 0 in $l_{2}$ will be the closed convex hull of the set $\left\{x \in l_{2}: x_{1}=0, \sum x_{i}^{2} \leqq 1\right\}$ together with the points $u=(1,1,0,0,0, \cdots)$, $v=(1,-1,0,0, \cdots)$ and their negatives. Using a theorem of V. L. Klee as in $[4, \S 3]$, we can construct a dense Hamel basis $B$ for $l_{2}$ which contains $x=(1,0,0, \cdots)$ and $y=(0,1,0,0, \cdots)$. Let $E$ be the linear span (not closed) of $B \sim\{y\}$, and let the unit ball $U$ of $E$ be $O \cap E$. Then the completion $\hat{E}$ of $E$ is $l_{2}$ with the norm induced by $\hat{U}$, and since $x=2^{-1}(u+v), x$ is not an extreme point of $\hat{U}$. It is an extreme point of $U$, however, since $y \notin E$ implies that $x$ is the only point of the segment $[u, v]$ which is in $E$, and (from the definition of 0 ) any segment in 0 which contains $x$ in its interior must lie in $[u, v]$.

Our Theorem 4 is not formulated in precisely the same manner as Buck's theorem [2, Theorem 2], where it was proved (for $E$ a real space and $M$ closed) in the following form: Let $k>0$ be an integer and let $E_{f}^{\boldsymbol{k}}=\left\{x \in E:\|x\|-f(x) \leqq k^{-1}\right\}$; then $f$ is an extreme point of $U^{*} \cap M^{\perp}$ if and only if $E=E_{f}^{\boldsymbol{k}}-E_{f}^{\boldsymbol{k}}+M$ for $k=1,2,3, \ldots$. This follows from Theorem 4 , however, upon observing that $E_{f}=k E_{f}^{k}$ for all $k>0$.

An elegant reformulation of Theorem 3 (and hence of Theorem 4) may be obtained (as in [2]) by setting $V_{f}^{\prime}=\bigcap_{1}^{\infty}\left(k^{-1} E_{f}-k^{-1} E_{f}+M\right)$; since $V_{f}^{\prime}=E$ if and only if $E_{f}-E_{f}+M=E, f$ is an extreme point of $U^{*} \cap M^{\perp}$ if and only if the closed subspace $V_{f}^{\prime}$ is all of $E$. This formulation does not hold under the minimal hypotheses of Theorem 2, however, since $V_{y}^{\prime}=\bigcap_{1}^{\infty}\left(k^{-1} F_{y}-k^{-1} F_{y}+M\right)$ may be a proper subspace of $F$ even though $F_{y}-F_{y}+M$ is $\sigma(F, G)$-dense in $F$. Indeed, we may take $F$ and $G$ to be $E^{*}$ and $E$, respectively, in the example described 
above. Letting $U^{*}$ be $C$ and $M=\{\phi\}$, we have shown that there exists an $x \in C^{0} \equiv U$ such that $x$ is an extreme point of $C^{0}$, so $F_{x}-F_{x}$ $\equiv E_{x}^{*}-E_{x}^{*}$ is $\sigma(F, G)$-dense in $F$. Since $Q x$ is not an extreme point of $U^{* *}$, however, there exists (as in the proof of Theorem 1) an element $z \in E^{* *} \sim\{\phi\}$ which is bounded on $E_{x}^{*}-E_{x}^{*}$; hence $z$ vanishes on $V_{x}^{\prime}$ and therefore $V_{x}^{\prime}$ is proper.

Suppose $E$ is a normed space, $M$ a closed subspace of $E$, and $f \in U^{*}$. Buck has suggested that (setting $\left.V_{f}=\bigcap_{1}^{\infty}\left(k^{-1} E_{f}-k^{-1} E_{f}\right)\right) V_{f}+M$ may always be dense in $V_{f}^{\prime}$. That this need not happen may be seen as follows:

Let $E$ be the space $c_{0}$ of real sequences converging to zero, with the supremum norm; then $E^{*}$ is the space $l_{1}$ of absolutely summable sequences. Let $f$ be the functional on $E$ corresponding to the sequence $\left(2^{-1}, 2^{-2}, 2^{-3}, \cdots\right) \in l_{1}$, and let $M$ be the closed subspace of $c_{0}$ consisting of all $x$ such that $\sum n^{-2} x_{n}=0$. We will show that $V_{f}+M$ is not dense in $V_{f}^{\prime}$. To this end, let (for $n=1,2,3, \cdots$ ) $u_{n}$ be the sequence in $l_{1}$ whose first $n$ terms are zero, and whose $k$ th term is $2^{-(k-1)}$ for $k>n$. Then $\left\|f \pm u_{n}\right\|=\left\|u_{n}\right\|=1$ and (as in the proof of Theorem 1) each $u_{n}$ is bounded on $E_{f}-E_{f}$ and hence vanishes on $V_{f}$. Thus, the elements $2^{n}\left(u_{n}-u_{n+1}\right)$ vanish on $V_{f}$; since (as is easily verified) these latter form a total set over $c_{0}$, we see that $V_{f}=\{\phi\}$.

Let $\delta^{n}$ be the sequence which is 1 in the $n$th place and zero elsewhere, and write $2^{n-1} \delta^{n}=\left(2^{n-1} \sum_{1}^{n} \delta^{i}\right)-\left(2^{n-1} \sum_{1}^{n-1} \delta^{i}\right)$. Thus, for each $n, 2^{n-1} \delta^{n}$ is the difference of two elements from $E_{f}$. We will show that the element $\delta^{1}$ is in $V_{f}^{1}=\bigcap k^{-1}\left(E_{f}-E_{f}+M\right)$ but not in $M+V_{f}$. The latter is obvious; we must show, then, that $k \delta^{1} \in E_{f}-E_{f}+M$ for each $k>0$. Given $k$, choose $n>0$ such that $2^{n-1} n^{-2} \geqq k$.Then $k \delta^{1}$ $=n^{2} k \delta^{n}+\left(k \delta^{1}-n^{2} k \delta^{n}\right)$. The first term is in the convex set $E_{f}-E_{f}$ (since $2^{n-1} \delta^{n} \in E_{f}-E_{f}$ and $n^{2} k \leqq 2^{n-1}$ ) while the second is clearly in $M$. We have shown that $M+V_{f}$ is a proper subspace of $V_{f}^{\prime}$; since $M=M+V_{f}$ is closed, it is not dense.

In looking for a non-reflexive Banach space which might answer the question posed at the beginning of this section, one naturally turns to either $C(X)$ ( $X$ compact Hausdorff) or $L_{1}$ of some measure space. It is an interesting exercise to verify the following fact: If $E$ is $C(X)$ or $L_{1}$ (over an arbitrary measure space) then the following is true: For $f \in U^{*}$ let $H_{f}=\{x \in E: f(x)=\|x\|\}$. Then $f$ is an extreme point of $U^{*}$ if and only if $E=H_{f}-H_{f}$. Furthermore (defining $H_{x}^{*}$ in the obvious way), $x \in U$ is an extreme point of $U$ if and only if $E^{*}=H_{x}^{*}-H_{x}^{*}$. (Representations of $E^{*}$ for the above spaces, as well as characterizations of the appropriate extreme points, may be found in $[3 ; 5]$.) 
These results say, in effect, that each extreme point of $U$ or $U^{*}$ is a "vertex"; in finite dimensional spaces this is true for any space whose unit ball is a (centrally symmetric) polyhedron.

Added in proof. K. de Leeuw has suggested and Y. Katznelson has proved that the answer to the question raised at the beginning of $\$ 4$ is negative for the Banach space of all complex valued functions which are analytic for $|z|<1$ and continuous for $|z| \leqq 1$, with the supremum norm.

\section{BiBLIOGRAPHY}

1. N. Bourbaki, Espaces vectoriels topologiques, Actualités Sci. Ind. no. 1229, Paris, Hermann, 1955, Chapter IV.

2. R. C. Buck, $A$ complete characterization for extreme functionals, Bull. Amer. Math. Soc. vol. 65 (1959) pp. 130-133.

3. M. M. Day, Normed linear spaces, Berlin, Springer, 1958.

4. R. R. Phelps, Subreflexive normed linear spaces, Arch. Math. vol. 8 (1957) pp. 444-450.

5. J. Schwartz, A note on the space $L_{p}^{*}$, Proc. Amer. Math. Soc. vol. 2 (1951) pp. 270-275.

The Institute for Advanced Study 